Whitfield, J. F. \& Appleyard, R. K. (1957). J. gen. Microbiol. 17, 453-466

\title{
Formation of the Vegetative Pool by Induced Defective and Healthy Lysogenic Strains of Escherichia coli
}

\author{
By J. F. WHITFIELD and R. K. APPLEYARD
}

Biology Division, Atomic Energy of Canada Ltd., Chalk River. Ontario, Canada

\begin{abstract}
SUMMARY: Comparison of two defective $\lambda$ prophages carried by strains of Escherichia coli, with respect to their physiological properties following induction, has revealed that one defective mutant $(\lambda i 2)$ can multiply normally as vegetative phage but cannot mature into normal infectious phage, whereas the other defective phage $(\lambda i 1)$ can undergo only limited multiplication since it is unstable in the vegetative state. Consideration of the properties of $\lambda i 1$ has led to an hypothesis that radiation induction causes the prophage to produce a vegetative replica which becomes autonomous rather than conversion of the prophage into vegetative phage.
\end{abstract}

Lysogenic strains of Escherichia coli containing defective forms of the bacteriophage $\lambda$ have been investigated by Appleyard (1954, 1956) and by Jacob \& Wollman (1956). They are distinguished by an almost total failure to liberate healthy phage following induction, and Appleyard (1956) showed that the defect may be transmitted by extracellular defective phage. The phage genes responsible for the defects resemble the recessive lethal genes of higher organisms; induction by ultraviolet irradiation of a doubly lysogenic strain, in which a normal and a defective prophage are present in the same cell, is followed by liberation at lysis of healthy phage particles, some of which have acquired genes from the defective prophage by recombination during the latent period.

As with recessive lethal genes, phage defects of independent origin would only rarely be expected to be genetically or physiologically identical. Appleyard \& Whitfield (1956) demonstrated recombination between two such defects to give rise to normal progeny. Therefore, they are not allelic but are genetically distinct factors. In the present study the physiological effects of these defects have been compared.

\section{METHODS}

\section{Bacteria and bacteriophages}

We used as experimental material strains of Escherichia coli and bacteriophages described in earlier papers from this laboratory (Appleyard, 1956; Appleyard, McGregor \& Baird, 1956). The two defects of independent origin chosen for investigation were those originally isolated in strains C33 $(\lambda i 2)$ and C60 ( $\lambda$ i1) (Appleyard, 1954). Both defects can be transmitted through extracellular phage to the same sensitive bacterium (Appleyard, 1956) with the advantage that observed physiological differences cannot then be due to strain differences between the bacterial hosts in which the defects were originally found. The cultures $\mathrm{C} 112(\lambda i 1)$ and $\mathrm{C} 112(\lambda i 2)$ as well as the healthy lysogen 
C112 $(\lambda s)$ used in our experiments were prepared by artificial lysogenization of the same sensitive strain C112, a descendant of strain 12-112 of Wollman (1953). Bacteriophage $\lambda-s$ was chosen as the healthy control phage, since both it and $\lambda i 1$ and $\lambda i 2$ were derived from the same ancestral strain of $E$. coli $\mathrm{K} 12(\lambda)$.

\section{Production of healthy phage by ultraviolet-stimulated reverse mutation}

If the defects arise as mutations of phage genes, many of them should be reversible through 'back mutation'. To study the frequency of u.v. stimulated reversion at various steps of phage development, a culture of defective lysogenic organisms was first induced at time zero by an exposure to a small dose of u.v. radiation. The induced organisms were allowed to develop and at various times during development several portions of the induced culture were exposed to different doses of u.v. radiation and after each dose the bacteria yielding healthy phage were counted. At any given time, the slope of the mutation/dose curve near the origin was taken as a measure of mutability. The detailed procedure was as follows.

Defective lysogens were prepared for induction by one of two methods:

(1) Organisms were grown on the surface of nutrient agar plates and, while still in the logarithmic phase of growth, were washed off in ice-cold buffer and diluted to a turbidity value (nephelometrically determined) which corresponded to a concentration of $3 \times 10^{8}$ viable organisms $/ \mathrm{ml}$.

(2) Organisms were grown in liquid Tryptone (Difco) broth with aeration to a concentration of $3 \times 10^{8}$ viable organisms $/ \mathrm{ml}$. (just out of the logarithmic phase of growth). They were then separated from the broth by centrifugation at $4^{\circ}$ for $3 \mathrm{~min}$. at $8000 \mathrm{~g}$ and resuspended in an equal volume of ice-cold buffer. In either case the organisms were at once exposed to u.v. radiation (2537A) for $15 \mathrm{sec}$. $\left(225 \mathrm{ergs} / \mathrm{mm}^{2}{ }^{2}\right)$ to induce them. Tryptone was added to a concentration of $1 \%(\mathrm{w} / \mathrm{v})(1 \mathrm{ml}$. of a $10 \%(\mathrm{w} / \mathrm{v})$ solution of Tryptone to $9 \mathrm{ml}$. suspension of organisms) and the culture was aerated at $37^{\circ}$.

At each time to be investigated, the organisms in $5 \mathrm{ml}$. of the culture were spun down and resuspended in an equal volume of ice-cold buffer as before. Separate $1 \mathrm{ml}$. portions of this buffer suspension were exposed to a further dose of u.v. radiation usually for 20,40 and 60 sec., and were at once assayed, with an unirradiated control, as plaque-formers before lysis by the agar layer technique.

\section{Recombinational analysis of pool size}

It is possible to superinfect an induced lysogenic organism with a phage which has been suitably marked genetically, and to relate the number of superinfecting normal phage/induced lysogenic organism and the proportion of recombinant phage released at lysis to the genetic or vegetative pool size (Visconti \& Delbrück, 1953) in the induced lysogenic organism at the time of superinfection. This relation will be described in full in the experimental section of the present paper. To determine the proportion of recombinants produced by superinfection following induction, cultures of $\mathrm{C} 112(\lambda s), \mathrm{C} 112$ 
$(\lambda i 1)$ and $\mathrm{C} 112(\lambda i 2)$ were grown to $3 \times 10^{8}$ viable organisms $/ \mathrm{ml}$. in Tryptone broth. Ten $\mathrm{ml}$. culture were spun down at $7000 \mathrm{~g}$. The organisms were resuspended in $9 \mathrm{ml}$. buffer and u.v.-irradiated (225 ergs $\left./ \mathrm{mm}^{2}{ }^{2}\right)$. One ml. of a $10 \%(\mathrm{w} / \mathrm{v})$ solution of Tryptone in distilled water was added to the induced culture, which was then aerated at $37^{\circ}$. After various time intervals, $0.9 \mathrm{ml}$. samples of the culture were removed and transferred to a tube containing $0 \cdot 1 \mathrm{ml}$. at various titres of phage $\lambda v 1 h$ (clear-plaque former) a host-range mutant of the weak virulent $\lambda$ phage (Appleyard, 1954; Appleyard et al. 1956). Beginning at any time between 0 and $45 \mathrm{~min}$. after induction of the lysogenic host organisms $99.9 \%$ of the phage $\lambda v 1 h$ was adsorbed within 15 min. at $37^{\circ}$. This fraction remained the same in mixtures containing up to 60 phage particles/organism.

Each infected culture was aerated until $30 \mathrm{~min}$. after the appearance of visible lysis. The lysates were then shaken with $\mathbf{0} \cdot \mathbf{1} \mathrm{ml}$. chloroform $/ \mathrm{ml}$. lysate, diluted 1/100 in broth and assayed by the double layer method on plates containing medium to a depth of not less than $1 \mathrm{~cm}$. (Jacob \& Wollman, 1954) to determine the numbers of turbid (recombinant) and clear plaques (normal parental phenotype).

In order to study changes in the normal healthy lysogenic strain of Escherichia coli $\mathrm{C} 112(\lambda s)$ following induction, a slightly different procedure was followed. The lysates were diluted and mixed with sufficient organisms from a freshly grown culture of the sensitive strain CR600 (Appleyard et al. 1956) to obtain a phage to bacterium ratio of at most $1: 100$. The infected culture was plated before lysis on the host-range indicator strain $\boldsymbol{E}$. coli CR63. This method of pre-adsorption eliminates any distortion of the data due to phenotypic mixing (Appleyard et al. 1956) as well as improving the clarity and size of the plaques. A phage to bacterium ratio of 1:100 was used to avoid double infection, which would also distort the results.

\section{RESULTS}

\section{Physiological comparisons between lysogens carrying defects i1 and i 2}

The defects show superficial similarities. Both are transferable by infection and can therefore be studied in bacteria having otherwise identical genotypes. Mass lysis of bacteria carrying either defective prophage can be induced by small doses of u.v. radiation. It occurs $85 \mathrm{~min}$. after exposure to u.v. light with liberation of an average of one healthy phage $/ 10^{5}$ to $10^{6}$ induced organisms. In both defective lysogens the production of infective phage, i.e. 'reverse mutants', can be stimulated by exposure to further doses of u.v. radiation during development, an average of 6 to 10 phage particles being released from each exposed organism.

\section{Differences in frequency of $u . v .-$ induced reversions of $\mathrm{i}$ to $\mathrm{i}^{+}$}

A marked difference between the two defects was observed with respect to frequency of reverse mutant phage detected following the double irradiation technique described above (Fig. 1). Following induction of organisms of 
Escherichia coli $\mathrm{C} 112(\lambda i 2)$ in the logarithmic phase of growth, the frequency of reversions was over 10 times as great when the second irradiation was applied at $30 \mathrm{~min}$. as when the second irradiation was applied at $0 \mathrm{~min}$. (Fig. 1A). After $30 \mathrm{~min}$., the frequency dropped sharply. In log phase organisms of $\mathrm{C} 112$ ( $\lambda i 1$ ), no such increase was observed (Fig. 1 A). Although the variation in time of the u.v. stimulation was somewhat different for older cultures (phase of retarded growth) the marked contrast in behaviour between $i 1$ and $i 2$ remained (Fig. 1 B). These observations suggested to us that the number of $\lambda i 2$ vegetative phage units increases with time following induction, whereas the number of $\lambda i 1$ vegetative phage units does not. To investigate this possibility it was necessary to devise ways of measuring the size of the vegetative or genetic pool in the induced defective and normal lysogens.

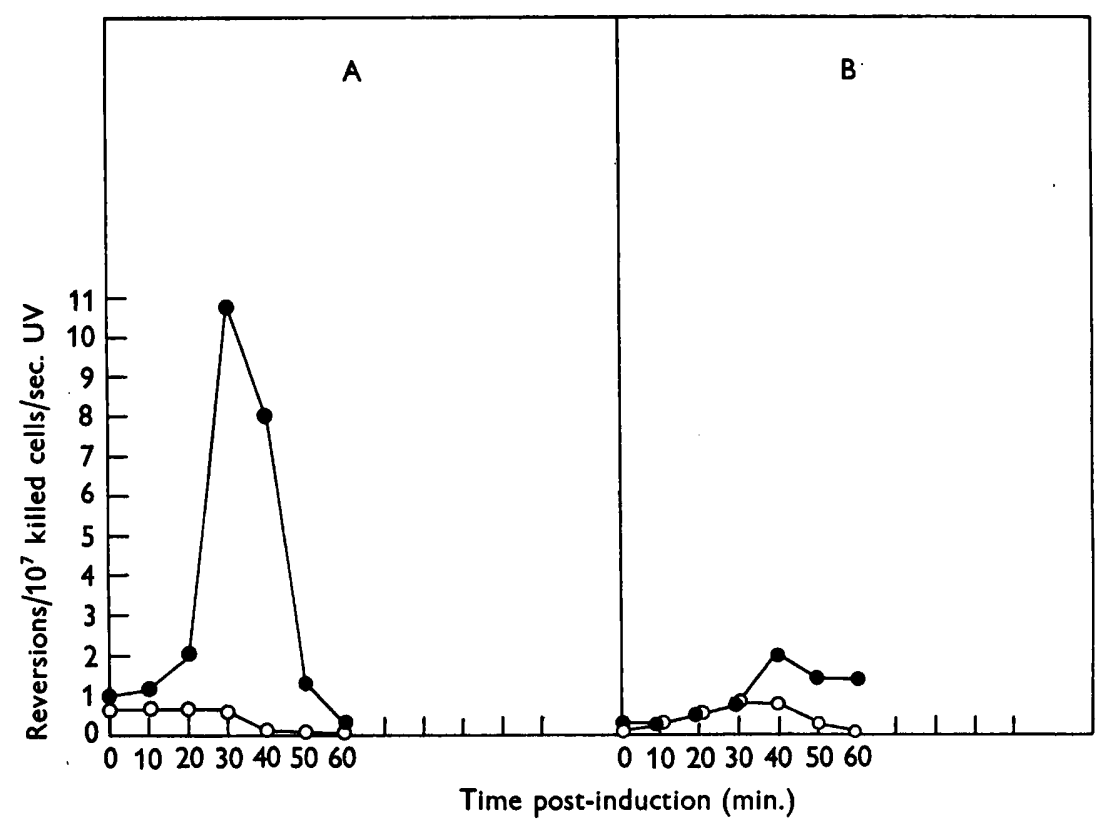

Fig. 1. The variation with time of the u.v. stimulated production of healthy phage following induction of Escherichia coli $\mathrm{C} 112(\lambda i 1)(\circ-0)$ and $E$. coli $\mathrm{C} 112(\lambda i 2)(\bullet-\bullet)$, (A) from cultures in the logarithmic phase of growth, (B) from cultures in the retarded phase of growth.

\section{Recombinational estimate of pool size}

Principles of experiment. By the genetic or vegetative pool we mean the total number of actively-recombining replicas of the phage genotype inside the organism (Visconti \& Delbrück, 1953). The simplest method of estimating the pool size is to measure the number of recombining units by some kind of superinfection with a genetically marked phage, and experiments on these lines have been carried out by a number of workers (Levinthal \& Visconti, 1953; Visconti \& Garen, 1953; Jacob \& Wollman, 1956). It is clear from the 
work of Jacob (1954), as well as from our own experiments, that the recombinational method includes the prophages in its measure of the pool size. It therefore strictly measures the genetic pool size rather than the vegetative. It was, however, necessary to avoid possible complications connected with selection for or against recombinants, with variations of the recombination process as a function either of time of irradiation (Jacob \& Wollman, 1955) or of the physiological state of the organisms in which defective development was proceeding. Moreover, we were unable to measure the total yield because it included defective phage. We therefore used a variation of the simple method based on the following argument.

Let $m$ be the number of defective, recombinable phage replicas/induced organism and let $n$ be the number of superinfecting healthy phage particles/ organism. If the ratio of $m$ to $n$ be constant throughout the remainder of the latent period, then the number of recombinants, $N$, issuing from such an infection should be directly proportional to the fraction of matings in which recombination is possible. If matings are at random, this fraction is $2 m n /(m+n)^{2}$.

Then,

$$
N=\frac{2 K m n}{(m+n)^{2}}
$$

Since the numbers of reciprocal recombinants are statistically equal in infections with $\lambda$ phage (Jacob \& Wollman, 1954; Wollman \& Jacob, 1954), the fraction of viable recombinants in the yield will be some constant fraction of $N$

$$
N^{\prime}=\frac{K^{\prime} m n}{(m+n)^{2}}
$$

whereas the total fraction of viable phage particles will be $n /(m+n)$.

Therefore, the proportion, $p$, of recombinants among the total yield of active phage particles will be

$$
p=\frac{K^{\prime} m}{(m+n)}
$$

(3) may be rewritten as a linear relation between the observable quantities $(n p)$ and $p$ :

$$
n p=-m p+K^{\prime} m
$$

By comparing different multiplicities of superinfection, $K^{\prime}$ may be eliminated

$$
\frac{p}{p^{\prime}}=\frac{m+n^{\prime}}{m+n} \text {. }
$$

To determine the pool size in induced defective lysogens, we studied recombination between the clear-plaque marker, $v 1$, and the defect $i 1$ or $i 2$ when the superinfecting phage was $v 1 i 1^{+} i 2^{+}$and the carried phage $v 1^{+} i 1 i 2^{+}$ or $v 1^{+} i 1^{+} i 2$; in such an experiment the only viable recombinant is $v 1^{+} i 1^{+} i 2^{+}$. The complementary recombinant $v 1 i 1$ or $v 1 i 2$ is not scorable. To determine the pool size in induced healthy lysogens, we studied recombination between the markers $v 1$ and $h$ when the superinfecting phage was $v 1 h$ and the carried 
phage was $v 1^{+} h^{+}$. Scoring only one recombinant class $v 1^{+} h$ among the total $h$ phage in this case is analogous to the method of estimating the pool size in defective lysogens.

Validity of the method. The linearity of the relation (4) between $(n p)$ and $p$ has been confirmed over a wide range of times and multiplicities of superinfection. A typical set of data is given in Table 1 and plotted as a test of linearity in Fig. 2. The straight lines derived from a number of other superinfections are plotted in Figs. 3 and 4.

Table 1. Calculation of the values ( $\mathrm{np}$ ) and $\mathrm{p}$ of text relation (4) using experimental data from superinfection of Escherichia coli $\mathrm{C} 112(\lambda i 2)$ with phage $\lambda v 1 i 2^{+} h 15$ min. after induction with u.v. light

The values $(n p)$ and $p$ are plotted in Fig. 2.

$\begin{array}{rcccc}n * & v 1^{+} i 2^{+} & v 1 i 2^{+} & p & n p \\ 1 \cdot 6 & 224 & 2763 & 0 \cdot 075 & 0 \cdot 110 \\ 4.7 & 221 & 3738 & 0 \cdot 055 & 0 \cdot 258 \\ 10 \cdot 0 & 115 & 3184 & 0 \cdot 035 & 0 \cdot 350 \\ 21 \cdot 2 & 140 & 6136 & 0 \cdot 021 & 0 \cdot 445\end{array}$

* In order to allow for the Poisson distribution of the infecting particles, the values of $n$ are calculated from the observed average multiplicity of infection, $x$, by the formula $n=x /\left(1-e^{-x}\right)$.

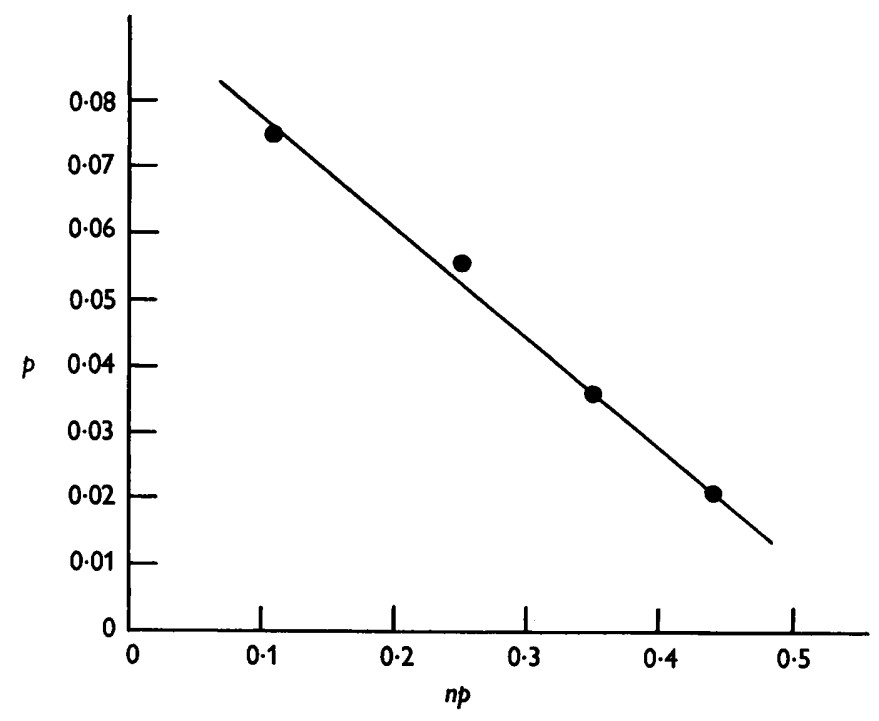

Fig. 2. Test of the linearity of relation (4) in the text between $(n p)$ and $p$. Data obtained from superinfection of Escherichia coli $\mathrm{C} 112(\lambda i 2)$ with $v 1 i 2^{+} h$ at $15 \mathrm{~min}$. after $\mathrm{u} . \mathrm{v}$. induction. The data and calculations are recorded in Table 1 . This line is also line $B$ of Fig. 4.

Such straight lines as that of Fig. 2 have confirmed the validity of the assumptions concerning random mating and absence of selection upon which our estimates of pool size are based. They show that very high multiplicities of superinfecting phage can enter the induced cell and take part in recombi- 
nation, in contrast to the situation in multiple infections of sensitive cells (Wollman \& Jacob, 1954). Further, any selection for or against the superinfecting phage during multiplication should be a function of the extent of that multiplication and so, inversely, of the multiplicity of superinfection: hence it would give rise to curvature of the lines which is not observed. Loss of a constant appreciable fraction of the superinfecting phage, for example during penetration of the induced cell, is not excluded by the linearity and could give rise to a constant factor of error in the estimates of pool size. However, this is unlikely, in view of the good agreement of the pool size estimated shortly after induction (see Fig. 2 and below) with the number of prophages per cell estimated by other methods.

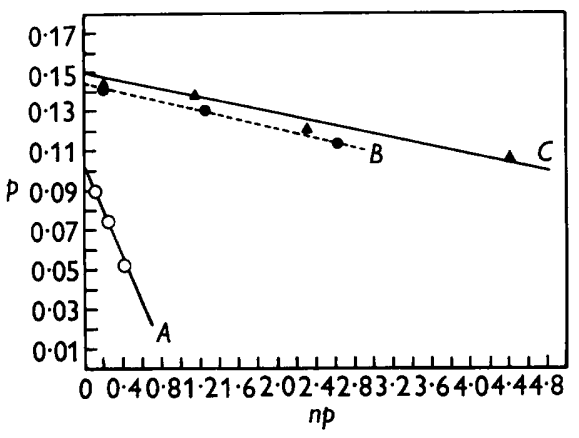

Fig. 3

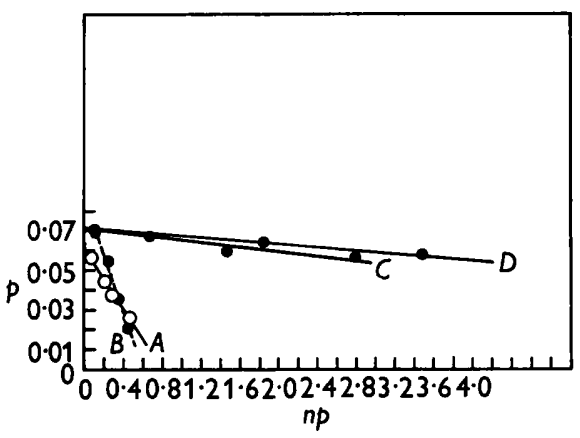

Fig. 4

Fig. 3. Determination of pool size $(m)$ by linear plots of relation (4) of text.

$A$ : Escherichia coli $\mathrm{C} 112(\lambda s)$ superinfected at $15 \mathrm{~min}$. after induction. Pool size = 8.8.

$B$ : $E$. coli $\mathrm{C} 112(\lambda s)$ superinfected at $40 \mathrm{~min}$. after induction. Pool size $=95 \cdot 4$.

$C: E$ coli $\operatorname{C112}(\lambda s)$ superinfected at $45 \mathrm{~min}$. after induction. Pool size $=\mathbf{9 5 \cdot 2}$.

Fig. 4. Determination of pool size $(m)$ by linear plots of relation (4) of text.

$A$ : Escherichia coli $\mathrm{C} 112(\lambda i 1)$ superinfected $43 \mathrm{~min}$. after induction. Pool size $=12 \cdot 4$. $B$ : $E$. coli $\mathrm{C} 112(\lambda i 2)$ superinfected 15 min. after induction. Pool size $=\mathbf{5 \cdot 9}$.

$C$ and $D$ : E. coli $\mathrm{C} 112(\lambda i 2)$ superinfected $45 \mathrm{~min}$. after induction. Average pool size $=201$.

\section{Measurements of pool size as a function of time}

We have used the recombinational analysis just described to follow the size of the genetic pools as a function of time in the defective lysogens Escherichia coli $\mathrm{C} 112(\lambda i 1), \mathrm{C} 112(\lambda i 2)$, and the healthy lysogen $E$. coli $\mathrm{C} 112(\lambda s)$. The results are shown in Figs. 5 and 6. The three strains behave differently and will be discussed in succession.

Healthy lysogen (Fig. 5, A). During the first $15 \mathrm{~min}$. after infection the number of recombinable particles remained nearly constant, but by $35 \mathrm{~min}$. it had increased from 8 to 20 . By $40 \mathrm{~min}$. the pool size had increased to 95 particles and there it remained up to $45 \mathrm{~min}$. This increase with time is reflected in the difference between the slope of line $A$ and lines $B$ and $C$ of Fig. 3. The observations of Weigle \& Delbrück (1951), that the first intracellular mature phage particles appeared between 40 and $45 \mathrm{~min}$. after induction have been shown to apply to lysogenic derivatives of Escherichia coli 
of $\mathrm{C} 112$ under the conditions of our experiments (Appleyard, unpublished). We therefore attribute the levelling off of the pool size to the onset of maturation.

Defective lysogen Escherichia coli $C 112(\lambda i 2)($ Fig. $5, B)$. The number of recombinable particles in induced $E$. coli $\mathrm{C} 112(\lambda i 2)$ at first remained constant and then rose steeply as in $E$. coli $\mathrm{C} 112(\lambda s)$. However, instead of reaching a plateau it continued to rise to about 201 particles, twice the value in the healthy strain, and we were not able to detect any levelling off at the latest times at which we were able to make measurements. The rate of multiplication of phage $\lambda i 2$ appeared to be about the same as that of phage $\lambda s$ (compare Fig. $5, A B$ with $5, C$ ). These results suggest that the maturation of $\lambda i 2$ is impaired whereas its vegetative multiplication is normal.

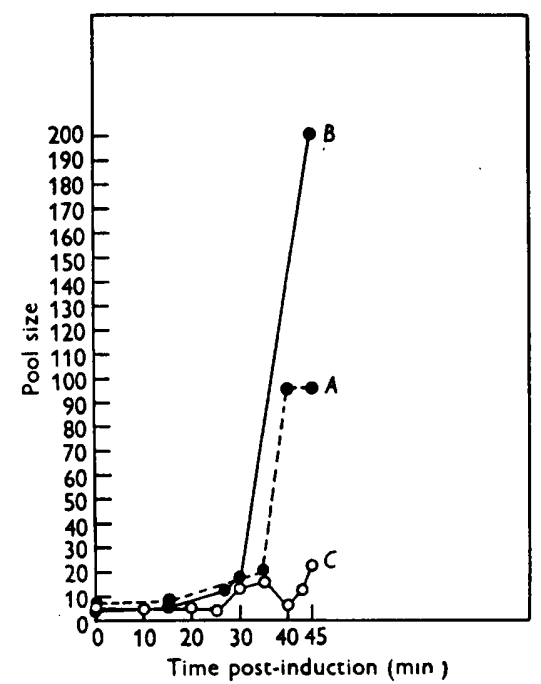

Fig. 5

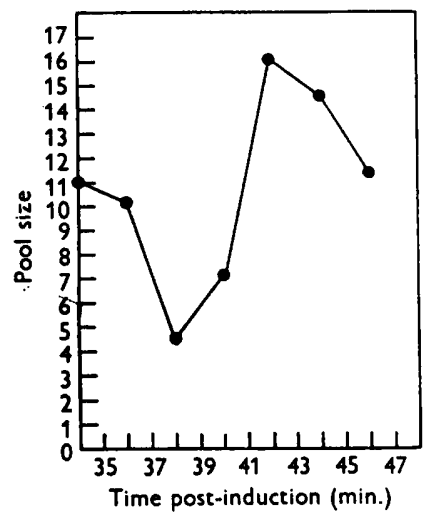

Fig. 6

Fig. 5. Variation of the pool size with time following induction. A: Escherichia coli $\mathrm{C} 112$ $(\lambda s)$. B: E. coli C112 ( $\lambda$ i2). C: E. coli $\mathrm{C} 112(\lambda i 1)$.

Fig. 6. Variation of the pool size with time following induction of Escherichia coli $\mathbf{C} 12$ $(\lambda i 1)$.

Defective lysogen Escherichia coli $C 112(\lambda i 1)$ (Figs. 5, $C$ and 6 and Table 2). There were marked differences between the events in E. coli C112 $(\lambda i 1)$ and those in $E$. coli $\mathrm{C} 112(\lambda i 2)$. The first multiplication of phage $\lambda i 1$ took place between 25 and 30 min. after induction but was followed by a decline in numbers between 30 and $40 \mathrm{~min}$. Two other experiments were performed to confirm this picture. In the second experiment the value of $m$ was 14.6 at $30 \mathrm{~min}$. and 16.3 at $35 \mathrm{~min}$; by $40 \mathrm{~min}$. this number had declined to 8.8 and at $45 \mathrm{~min} . m$ had increased to $21 \cdot 1$. The results of these two experiments have been combined and presented in Fig. 5, $C$ and Table 2.

Further analysis of the events described in the preceding paragraph was carried out. Samples of an induced culture of Escherichia coli $\mathrm{C} 112(\lambda i 1)$ 
were superinfected at $2 \mathrm{~min}$. intervals from 34 to $46 \mathrm{~min}$. From the data presented in Fig. 6 it may be seen that as in the first two experiments the value of $m$ between 34 and $36 \mathrm{~min}$. was constant, or nearly so, at 10. However, by $38 \mathrm{~min}$. $m$ had decreased to $4 \cdot 5$, being followed by the usual rise, which in this case began between 40 and $42 \mathrm{~min}$.

Table 2. Calculation of the pool size $(m)$ in cells of Escherichia coli $\mathrm{C} 112(\lambda i 1)$ superinfected with $\lambda$ v1 $i 1^{+} h$ at various times post-induction

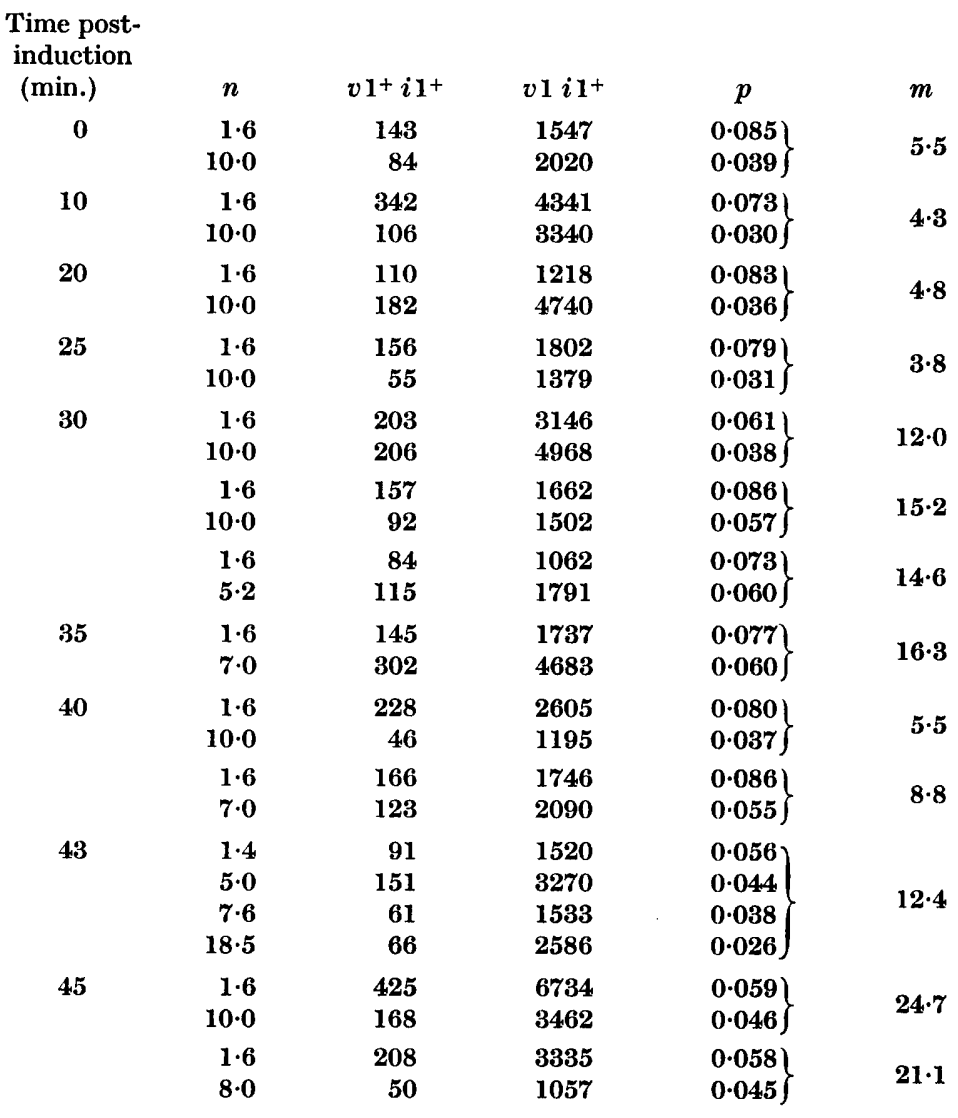

The difference between the pool sizes at $\mathbf{4 3 - 4 5}$ min. after induction of Escherichia coli $\mathrm{C} 112(\lambda i 1)$ and $E$. coli $\mathrm{C} 112(\lambda i 2)$ is reflected in the difference between the slope of line $A$ and those of lines $C$ and $D$ of Fig. 4. It appears that phage $\lambda i 1$ can undergo only limited multiplication in the vegetative state.

\section{DISCUSSION}

Measurements of pool size and u.v.-stimulated liberation of healthy phage show that defects $i 1$ and $i 2$ bring about different physiological changes following induction. In particular, estimates of genetic pool size show that 
phage $\lambda i 1$, unlike phage $\lambda i 2$, experiences some difficulty in vegetative multiplication as was suggested by the curves of u.v.-stimulated reversions (Fig. 1, A). Comparison of the pool formed by each defective phage with that formed by healthy phage (Fig. 5) permits a more detailed interpretation of the nature of both defects.

The nature of defect $i 2$. The characteristic difference between phage $\lambda i 2$ and phage $\lambda s$ is the failure of the number of recombinable particles in the case of the induced $i 2$ lysogen to stop rising and reach a plateau. Although the time at which the rapid increase in recombinable particles took place in Escherichia coli $\mathrm{C} 112(\lambda i 2)$ was 5 min. earlier than that in $E$. coli $\mathrm{C} 112(\lambda s)$, this is more likely to be correlated with later lysis of induced E. $\operatorname{coli} \mathbf{C} 112(\lambda s)$, than to be a characteristic of phage $\lambda i 2$.

Weigle \& Delbrück (1951) observed that the first mature infectious phages appeared in cells of induced healthy Escherichia coli $\mathrm{K} 12(\lambda)$ between 40 and $45 \mathrm{~min}$. after induction. This observation has been confirmed for our strains (Appleyard, unpublished). If the attainment of a constant pool size in the induced normal lysogenic organism, as in coliphage T2- or T4-infected organisms, is due to the intervention of maturation, then we can describe the $i 2$ defect as a failure to undergo the first step of maturation, i.e. removal from the vegetative pool.

How much of the machinery of maturation can be set up by phage $\lambda i 2$ remains unknown. However, Dr E. Kellenberger (personal communication) observed that empty phage heads and tails both appeared in lysates after induction of the strain C33 $(\lambda i 2)$. Whitfield, Appleyard \& Baird (unpublished) prepared doubly lysogenic strains of Escherichia coli $\mathrm{C} 112$ which carried both phage $\lambda i 2$ and a host range $(h)$ mutant of normal phage $\lambda$. Among the phage in lysates from such u.v.-induced strains, they observed healthy phage which were genotypically $h$ but which possessed $h^{+}$coats derived from the defective phage. Possession of an $h$ coat permits the individual phage to absorb on to the host range indicator strain $E$. coli CR63 while a phage particle with an $h^{+}$coat cannot absorb on to this strain (Appleyard et al. 1956). Therefore, it seems that phage $\lambda i 2$ can initiate construction of and at least in part control the specificity of the morphological components required for maturation.

Another defective $\lambda$ phage was studied by Jacob \& Wollman (1956). They concluded that their defect prevents maturation of vegetative $\lambda$ phage. Since these authors were unable to demonstrate the presence of mature defective phage even after superinfection with healthy phage, they suggested that each defective vegetative unit interferes with its own maturation. However, in the presence of healthy phage, $\lambda i 2$ can be matured (Appleyard, 1956). We therefore conclude that it belongs to a different class of maturation defects.

The nature of $i 1$. In the prophage state, phage $\lambda i 1$ is stable, for it can be transmitted through many cell generations without loss. In induced and spontaneous lysates from the double lysogenic strain Escherichia coli $\mathrm{C} 112$ $(\lambda i 1, \lambda \mathrm{Cl} h)$, the major part of the progeny population is genotypically $h$ but phenotypically $h^{+}$. It would appear from this that phage $\lambda i 1$, as is the case 
with phage $\lambda i 2$, can direct the synthesis of those elements which confer upon the mature virus its host range properties (Whitfield, Appleyard \& Baird, unpublished).

In the vegetative state, phage $\lambda i 1$ can multiply, since two successive bursts of multiplication are observed to follow induction. However, these two events are separated by an interval of decrease in the number of vegetative particles. Lambda- $i 1$ phage is therefore unstable in the vegetative state. The present experiments do not enable us to distinguish clearly between a genuine physico-chemical instability of vegetative phage $\lambda i 1$ and a cessation of multiplication coupled with some hypothetical normal process of removal from the vegetative pool. However, removal could not in this case be through a normal maturation process, as it begins too soon.

Some support for believing vegetative phage $\lambda i 1$ to be unstable is provided by the observations of Appleyard \& Whitfield (1956). They observed that superinfection immediately following u.v. irradiation of cultures of Escherichia coli $\mathrm{C} 112(\lambda i 2)$ with phage $\lambda i 1$ resulted in the appearance of no healthy recombinant phage-yielding organisms. Superinfection at later times produced increasing numbers of normal phage yielders. In the opposite situation, with phage $\lambda i 2$ and $E$. coli $\mathrm{C} 112(\lambda i 1)$, superinfection immediately following u.v. irradiation resulted in the appearance of nearly the maximum number of normal phage-yielders possible under the conditions of the experiment. These observations suggest that phage $\lambda i 1$, unable to maintain its integrity in the host cytoplasm, would have to enter the host at a later time in order to be present in an intact state to participate in recombination with phage $\lambda i 2$ when that phage begins its vegetative multiplication.

Analyses of the deoxyribonucleic acid content of Escherichia coli $\mathrm{C} 112$ $(\lambda i 1)$ cultures at various times after u.v.-induction indicate that the DNA content remains constant during the first $\mathbf{3 0} \mathrm{min}$. after induction but shortly thereafter increases linearly at the same rate as that in induced normal lysogenic cultures of $E$. coli $\operatorname{C112}(\lambda s)$ (Whitfield, unpublished). It should be pointed out that the time of resumption of DNA synthesis following induction of these strains correlates well with the time at which the values of $m$ begin to rise and also with the time at which the frequency of u.v.-stimulated reversions sharply increases in $E$. coli $\mathrm{C} 112(\lambda i 2)$. These biochemical studies, to be published later, were carried out under the same conditions as the poolsize estimations.

If the instability of phage $\lambda i 1$ be due to extensive fragmentation resulting from the operation of some factor such as deoxyribonuclease, then large amounts of DNA fragments would appear in the acid-soluble nucleotide fractions from $\mathbf{3 0} \mathrm{min}$. to lysis of the culture. This was not observed. However, limited fragmentation of the vegetative particles remains a possibility.

During the decline the number of recombinable particles decreases not to zero, but to approximately the number preceding the first burst of multiplication; thus there seems to be a small persisting number of stable particles. We suggest that this persistent fraction represents the original prophages of the cell which by continued association with the bacterial nuclear material 
retain their stability. This hypothesis implies that in the process of induction, prophage is not itself physically converted to vegetative phage, for all the intracellular particles would then be unstable, but makes at least one vegetative replica which initiates vegetative multiplication. In the case of the unstable phage $\lambda i 1$, because the picture is not obliterated by large numbers of vegetative phage units, it can be seen to initiate two such attempts at vegetative multiplication, separated from each other and from the moment of induction by very approximately the division time of the un-irradiated lysogenic organisms.

The nature of $u . v$. radiation induction. We therefore propose the following hypothesis concerning the mechanism of u.v. radiation induction. The effect of the radiation is to modify the cell's nuclear apparatus in such a way that replicas formed by the prophage at its subsequent divisions become vegetative phage. It is of interest to relate this hypothesis in detail to a number of established facts. The work of Lwoff and his colleagues (Lwoff, 1953b) suggests the intervention of physiological processes in the induction process. Direct absorption of radiation energy by prophage is not needed to accomplish induction. We suggest that the effect of the radiation upon the nuclear apparatus is somewhat analogous to the mitotic delay well known as an effect of radiation upon the cells of higher organisms. DNA synthesis in both lysogenic and non-lysogenic microbial cells is inhibited by small doses of u.v. radiation (Siminovitch \& Rapkine, 1951; Kelner, 1953; Barner \& Cohen, 1956; Whitfield, unpublished), although various intermediates of DNA synthesis including deoxyribonucleotides appear as accumulated products in the medium (Kanazir, 1954; Kanazir \& Errera, 1954). Such changes would prevent the multiplication of the bacterial nuclear apparatus. If the prophage DNA were not similarly affected, the fresh replica would find itself in a predominantly cytoplasmic milieu. The availability of accumulated DNA products would permit it to multiply as an autonomous unit. The unbalanced condition of the cell would thus greatly favour vegetative multiplication. Similar hypotheses have been proposed by Jacob \& Stent (cited by Lwoff, $1953 a$ ).

Morse, Lederberg \& Lederberg (1956) observed that in Escherichia coli transduction of gal markers by $\lambda$ phage was effective only when the phage was obtained by induction of a lysogenic strain, and is not observed when the phage issued from a lytic infection. Their findings are most naturally explained if vegetative $\lambda$ phage does not become attached to galactose loci but after induction the linkage of some units to gal markers which they initially had as prophages persists, as does the associated stability in our experiments.

The number of prophages/organism. Bertani (1953) and Jacob (1954) were able to obtain approximate calculations of the number of prophages/lysogenic bacterium. Both workers (Bertani with lysogenic Shigella dysenteriae and Jacob with lysogenic Pseudomonas aeruginosa) concluded that the number of prophages/bacterium was close to the number of nuclei. The present studies lead to the same conclusion. The number of prophages can be inferred from the number of recombinable particles at zero minutes after induction. This 
number ranges from 4.6 to 6.0 with an average of about 5 and so closely approximates the average number of nuclei/cell in the logarithmic phase of growth (Whitfield, unpublished).

The authors would like to express their gratitude to Mr K. M. Baird for his assistance in carrying out some of the experiments recorded in this paper.

\section{REFERENCES}

Appleyard, R. K. (1954). Segregation of new lysogenic types during growth of a doubly lysogenic strain derived from Escherichia coli K12. Genetics, 39, 440.

Appleyard, R. K. (1956). The transfer of defective lambda lysogeny between strains of Escherichia coli. J. gen. Microbiol. 14, 573.

Appleyard, R. K., McGregor, J. F. \& Baird, K. M. (1956). Mutation to extended host range and the occurrence of phenotypic mixing in the temperate coliphage lambda. Virology, 2, 565.

Appleyard, R. K. \& Whitfield, J. F. (1956). Hereditary defects of temperate bacteriophage. Nature, Lond., 178, 1235.

Barner, H. D. \& Cohen, S. S. (1956). The relation of growth to the lethal damage induced by ultraviolet irradiation in Escherichia coli. J. Bact. 71, 149.

Bertani, G. (1953). Lysogenic versus lytic cycle of phage multiplication. Cold Spr. Harb. Symp. quant. Biol. 18, 65.

$\mathrm{J}_{\mathrm{ACOB}}, \mathrm{F}$. (1954). Les bactéries lysogènes et la notion de provirus. Paris: Masson et Cie.

JacoB, F. \& Wollman, E. L. (1954). Étude génétique d'un bactériophage tempéré d'Escherichia coli. I. Le système génétique du bactériophage $\lambda$. Ann. Inst. Pasteur, 87, 653.

JacoB, F. \& Wollman, E. L. (1955). Étude génétique d'un bactériophage tempéré d'Escherichia coli. III. Effect du rayonnement ultraviolet sur la recombinaison génétique. Ann. Inst. Pasteur, 88, 724.

$\mathrm{J}_{\text {ACOB }}, \mathrm{F}$. \& Wollman, E. L. (1956). Recherches sur les bactéries lysogènes defectives. I. Déterminisme génétique de la morphogenèse chez un bactériophage tempéré. Ann. Inst. Pasteur, 90, 282.

Kanazir, D. (1954). Accumulation de l'acide thymidylique chez $E$. coli. après irradiation UV. Biochim. biophys. Acta, 13, 587.

Kanazir, D. \& Errera, M. (1954). Métabolisme des acides nucléiques chez $\boldsymbol{E}$. coli $\mathbf{B}$ après irradiation ultraviolette. Biochim. biophys. Acta, 41, 62.

Kelner, A. (1953). Growth, respiration, and nucleic acid synthesis in ultravioletirradiated and in photoreactivated Escherichia coli. J. Bact. 65, 252.

Levinthal, C. \& Visconti, N. (1953). Growth and recombination in bacterial viruses. Genetics, 38, 500.

Lwoff, A. (1953a). L'induction. Ann. Inst. Pasteur, 84, 225.

LwoFf, A. (1953b). Lysogeny. Bact. Rev. 17, 269.

Morse, M. L., Lederberg, E. M. \& Lederberg, J. (1956). Transduction in Escherichia coli K12. Genetics, 41, 142.

Siminovitch, L. \& Rapkine, S. (1951). Modifications biochimiques au cours du développement des bactériophages chez une bactérie lysogène. C.R. Acad. Sci., Paris, 232, 1603.

Visconti, N. \& Delbrück, M. (1953). The mechanism of genetic recombination in phage. Genetics, 38. 5.

Visconti, N. \& Garen, A. (1953). Unity of the vegetative pool in phage-infected bacteria. Proc. nat. Acad. Sci., Wash. 39, 620. 
Weigle, J. J. \& Delbrück, M. (1951). Mutual exclusion between an infecting phage and a carried phage. J. Bact. 62, 301.

Wollman, E. L. (1953). Sur le déterminisme génétique de la lysogénie. Ann. Inst. Pasteur, 84, 281.

Wollman, E. L. \& JACoB, F. (1954). Étude génétique d'un bactériophage tempéré d'Escherichia coli. II. Mécanisme de la recombinaison génétique. Ann. Inst. Pasteur, 87, 674.

(Received 8 April 1957) 